\title{
Projective Fourier Analysis for Patterns
}

\author{
Jacek Turski \\ Department of Computer and Mathematical Sciences \\ University of Houston - Downtown
}

Pattern Recognition 33, 2033-2043, 2000

\begin{abstract}
Identifying $\mathbf{P S L}(2, \mathbb{C})$ as a projective group for patterns in the conformal camera model, the projective harmonic analysis on its double covering group $\mathbf{S L}(2, \mathbb{C})$ is presented in the noncompact and compact pictures - the pictures used to study different aspects of irreducible unitary representations of semisimple Lie groups. Bypassing technicalities of representation theory, but stressing the motivation and similarities with Euclidean Fourier analysis, each constructed picture of the projective Fourier analysis includes the Fourier transform, Plancherel's theorem and convolution property. Projectively covariant characteristics of the analysis in the noncompact picture allow rendering any of image projective transformations of a pattern (after removing conformal distortions) by using only one projective Fourier transform of the original pattern, what is demonstrated in a computer simulation. The convolution properties in both pictures must by used to develop algorithms for projectively-invariant matching of patterns. Work in progress on fast algorithms for computing with projective Fourier transforms and for rendering image projective transformations is discussed. Efficient computations of the convolutions would follow from the both fast projective Fourier transforms and their inverses.

KEYWORDS: Conformal camera, projectively invariant pattern classification, projective Fourier analysis, projectively covariant pattern representation.
\end{abstract}




\section{Introduction}

A visual system for image understanding and pattern analysis should be built upon some data structure representing visual inputs and algorithms storing and processing visual information which are well adapted to image transformations produced by different perspectives between objects and the imaging system. However, the most frequently used such data structures are based upon Fourier or wavelet analyses and, therefore, they are lacking projectively covariant characteristics. For example, although one can reconstruct a pattern that is rotated and translated in an image plane using only one Fourier transform of the original pattern, when image projective transformations are applied, this is no longer feasible.

Motivated by this deficiency, persisting in spite of substantial work, see $[1,2,3,4,5,6,7,8,9]$, see also [10] for a more general context, we have developed the projective analogue of Fourier analysis for patterns $[11,12,13]$. This Fourier analysis has been constructed upon the semisimple Lie group $\mathbf{S L}(2, \mathbb{C})$, the double cover of the projective group for patterns $\operatorname{PSL}(2, \mathbb{C})$, employing the mathematical framework (group representation theory) of Fourier analysis on groups [14]. It has been done in the noncompact picture [11] and the compact picture $[13,15]$, both pictures used to study irreducible representations of semisimple Lie groups [16]. Also, the discrete projective Fourier transform in the noncompact picture has been developed [12].

In this article we present an exposition of both pictures of projective Fourier analysis based on the similarity with Euclidean Fourier analysis; each picture is complementing the other in the same way the classical Fourier analysis and spherical Fourier analysis complements each other in making up Euclidean Fourier analysis. It provides a more intuitive exposition of this highly abstract and technical subject. For the precise definitions and mathematical proofs of results on projective Fourier analysis we refer to [11, 13] where also detailed expositions of the related projective geometry have been given.

We demonstrate (carefully avoiding technicalities but at the same time giving enough mathematical background) that in each picture the inverse projective Fourier transform decomposes the pattern's intensity function in

terms of the irreducible unitary representations of the related subgroup of the projective group, with the coefficients given by the corresponding Fourier transform. Because irreducible representations are the simplest transforma- 
tions that mirror the corresponding subgroup operation, the analysis possesses projectively covariant characteristics, which should be efficiently implemented. In fact, we discuss the author's work in progress on numerical implementations of the analysis for problems in projectively covariant image processing. The inverse projective Fourier transform in noncompact picture allows the reconstruction of any projective distortions of a pattern from only one projective Fourier transform of the original (undistorted) pattern, what is demonstrated in a computational test. Moreover, the convolution properties in both pictures are needed to develop projectively invariant pattern matching. It follows from the fact that the convolution in the noncompact picture is defined over the subgroup consisting of translations in the optical axis direction and rotations in the image plane of a camera, whereas the convolution in the compact picture is defined over the subgroup of all rotations of a camera, and both subgroups together generate the projective group.

The paper is organized as follows. The next section reviews the projective camera model which identifies the projective group for patterns and provides their projectively-invariant classification. In Section 3 the projective harmonic analysis in noncompact picture is presented which is followed in Section 4 by the development of the discrete projective Fourier transform in this picture. Next, in Section 5 the compact picture of the projective harmonic analysis is constructed. Section 6 contains discussions of the conformal camera model upon which the projective harmonic analysis is developed, its applications to image processing problems, and computer simulations of the image perspective transformations of a ring pattern. Also, this section presents some preliminary results on developing fast algorithms for computations with projective Fourier transforms and for rendering image projective transformations of patterns. The last section summarizes the paper.

\section{The projective group for patterns}

\subsection{The $P S L(2, \mathbb{C})$-camera}

The pinhole, or optical center, of a camera is located at the center of a planar projection. This is the point where the incoming rays of light intersect each other, giving images on an image plane. We let the plane $x_{2}=1$ in $\mathbb{R}^{3}=\left\{\left(x_{1}, x_{2}, x_{3}\right)^{t} \mid x_{i} \in \mathbb{R}\right\}$ be the image plane and the optical center be 
located at the origin. (Here " $t$ " stands for the transpose.) That is, the image points are given by the projection $j: \mathbb{R}^{3} \rightarrow \mathbb{C}$,

$$
j\left(\left(x_{1}, x_{2}, x_{3}\right)^{t}\right)=\frac{x_{3}+i x_{1}}{x_{2}}
$$

where in the image plane we have identified points $\left(x_{1}, 1, x_{3}\right)^{t}$ with complex numbers $z=x_{3}+i x_{1}$.

A pattern as a planar object "lives" on the image plane, the image projective transformations of which are generated by translating the pattern or rotating its projection on the unit sphere $\mathbf{S}_{(0,1,0)}^{2}$ centered at $(0,1,0)$ to form its "virtual" position, and then projecting them by $j$ in (1) back into the image plane, and by all finite iterations of these basic distortions, see Figure 1.

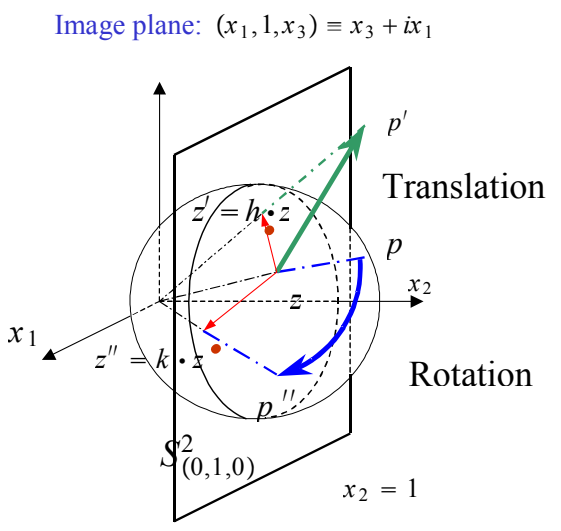

Fig. 1. Image projective transformations generated by translations and rotations in the camera model upon which the projective Fourier analysis is constructed. Note that rotations introduce conformal distortions; see the text for a full discussion. 
The choice of the unit sphere $\mathbf{S}_{(0,1,0)}^{2}$ is implied by the geometry of the camera, i.e., the choice of the image plane and the optical center.

In order to find the projective group generated in this way, we start by choosing Euler's angles $\left(\psi, \phi, \psi^{\prime}\right)$ where $\psi$ rotates about the $x_{2}$-axis followed by the angle $\phi$ rotation about the direction of the $x_{3}$-axis, and finally the angle $\psi^{\prime}$ rotation again about the rotated $x_{2}$-axis. We have shown in [11] that for rotations $R=R\left(\psi, \phi, \psi^{\prime}\right) \in \mathbf{S O}(3)$ acting on $\mathbf{S}_{(0,1,0)}^{2}$, there are two elements $k \in \mathbf{S U}(2) \subset \mathbf{S L}(2, \mathbb{C})$ given by

$$
k= \pm\left(\begin{array}{cc}
\cos \left(\frac{\phi}{2}\right) e^{-i\left(\psi+\psi^{\prime}\right) / 2} & i \sin \left(\frac{\phi}{2}\right) e^{-i\left(\psi-\psi^{\prime}\right) / 2} \\
i \sin \left(\frac{\phi}{2}\right) e^{i\left(\psi-\psi^{\prime}\right) / 2} & \cos \left(\frac{\phi}{2}\right) e^{i\left(\psi+\psi^{\prime}\right) / 2}
\end{array}\right)
$$

such that $j \circ R \circ\left(\left.j\right|_{\mathbf{S}_{(0,1,0)}^{2}}\right)^{-1}=k$ and $k$ 's are acting on the image plane $\mathbb{C}$ by linear-fractional transformations

$$
k \cdot z=\frac{\cos \left(\frac{\phi}{2}\right) e^{i\left(\psi+\psi^{\prime}\right) / 2} z+i \sin \left(\frac{\phi}{2}\right) e^{i\left(\psi-\psi^{\prime}\right) / 2}}{i \sin \left(\frac{\phi}{2}\right) e^{-i\left(\psi-\psi^{\prime}\right) / 2} z+\cos \left(\frac{\phi}{2}\right) e^{-i\left(\psi+\psi^{\prime}\right) / 2}} .
$$

Here, $\mathbf{S L}(2, \mathbb{C})$ is the group of $2 \times 2$ complex matrices of determinant one and $\mathbf{S U}(2)$ is its compact subgroup of matrices of the form $\left(\begin{array}{cc}\bar{\alpha} & \bar{\beta} \\ -\beta & \alpha\end{array}\right)$. Also, "o" denotes the composition of maps.

Similarly, there is a one-to-two correspondence between almost all translations $b \in \mathbb{R}^{3}$ of image points $x$ and the elements $h \in \mathbf{S L}(2, \mathbb{C})$ of the form $\left(\begin{array}{cc}\alpha & 0 \\ \gamma & \alpha^{-1}\end{array}\right), \gamma \in \mathbb{C}$ and $\alpha=\delta$ or $\alpha=i \delta$ with $\delta>0$, such that one has $j(x+b)=h \cdot j(x)$ where $h$ is acting on $\mathbb{C}$ by the linear-fractional transformation $h \cdot z=\left(\alpha^{-1} z+\gamma\right) / \alpha$. In fact,

$$
h= \pm\left(\begin{array}{cc}
\left(1+b_{2}\right)^{1 / 2} & 0 \\
\left(b_{3}+i b_{1}\right)\left(1+b_{2}\right)^{-1 / 2} & \left(1+b_{2}\right)^{-1 / 2}
\end{array}\right)
$$

if and only if $b=\left(b_{1}, b_{2}, b_{3}\right)^{t}$ with $b_{2} \neq-1$. It implies the factorizations: $h \in \mathbf{A} \overline{\mathbf{N}}$ if $1+b_{2}>0$ and $h=\varepsilon \mathbf{A} \overline{\mathbf{N}}$ if $1+b_{2}<0$, where $\overline{\mathbf{N}}=\left\{\left(\begin{array}{ll}1 & 0 \\ \gamma & 1\end{array}\right) \mid \gamma \in \mathbb{C}\right\}, \mathbf{A}=\left\{\left(\begin{array}{cc}\delta & 0 \\ 0 & \delta^{-1}\end{array}\right) \mid \delta \in \mathbb{R}_{+}\right\}, \varepsilon=\left(\begin{array}{cc}-i & 0 \\ 0 & i\end{array}\right)$.

From the discussion given above, it follows that the subgroup $\mathbf{S U}(2)$ acting by linear-fractional transformations on the image plane $\mathbb{C}$ represents 
image projective transformations of patterns (plus conformal distortions, see the end of this subsection) produced by rotations in a camera, with the maximal torus in $\mathbf{S U}(2), \mathbf{M}=\left\{\left(\begin{array}{cc}e^{i \psi} & 0 \\ 0 & e^{-i \psi}\end{array}\right)\right\}$, representing rotations in the image plane. Further, the subgroups $\mathbf{A}$ and $\varepsilon \mathbf{A}$ represent image transformations produced by translations of a camera in the direction of its optical axis.

In conclusion, the factorization $\mathbf{S L}(2, \mathbb{C})=\mathbf{S U}(2) \mathbf{A S U}(2)$, see [16], and the fact that both $\pm k$ (and $\pm h$ ) have the same action by linear-fractional transformations, imply that the projective group for patterns is the group $\operatorname{PSL}(2, \mathbb{C})=\mathbf{S L}(2, \mathbb{C}) /\{ \pm \mathbf{I}\}$. This is the quotient group of $\mathbf{S L}(2, \mathbb{C})$ obtained by identifying matrices that differ by sign.

Thus, for given patterns

$$
P=\{f: D \rightarrow \mathbb{R}\}
$$

their orbits

$$
\operatorname{PSL}(2, \mathbb{C}) \ni g \longmapsto P_{g}=\left\{f \circ g^{-1}: g D \rightarrow \mathbb{R}\right\}
$$

provide projectively-invariant classification of images as each orbit contains almost all perspectively distorted patterns such that two patterns that are on different orbits are not related by a perspective transformation. We refer to this camera model as the $\mathbf{P S L}(2, \mathbb{C})$-camera.

Although $P_{g}$ "inherits" the intensity of $P$ at the corresponding points, i.e., $f^{\prime}\left(\xi^{\prime}\right)=f(\xi)$ where $\xi^{\prime}=g \cdot \xi$ and $f^{\prime}=f \circ g^{-1}$, this camera model exhibits a "conformal lens optics". Therefore, this projective camera model is referred to also as the conformal camera. We proceed next to verify this claim but its comprehensive discussion is postponed until Section 6. To this end, we recall that a pattern on the image plane is first projected on the sphere $\mathbf{S}_{(0,1,0)}^{2}$, which is next rotated by the action of $\mathbf{S O}(3)$ and finally is projected back on the image plane, giving the image perspective transformation: $j \circ R \circ\left(\left.j\right|_{\mathbf{S}_{(0,1,0)}^{2}}\right)^{-1}(z)=k \cdot z$, cf., Figure 1. This image transformation of a pattern is conformal (i.e., it preserves angles) since the linear-fractional transformations are conformal. In conclusion, this camera model while being a useful starting point, needs a calibration to remove these conformal distortions. This is discussed in Section 6. 


\subsection{Projective Fourier analysis}

Any point of the image plane can be identified with the point of $\mathbb{C}^{2}=$ $\left\{\left(\begin{array}{l}z_{1} \\ z_{2}\end{array}\right) \mid z_{1}=x_{2}+i y, z_{2}=x_{3}+i x_{1}\right\}$ where the complex line $z_{2}=\mu z_{1}$ intersects the line $z_{1}=1$. Thus, the point $x_{3}+i x_{1}$ of the image plane is identified with the slope $\mu$ of the line. We note that the complex line $x_{3}+i x_{1}=\left(\mu_{1}+i \mu_{2}\right)\left(x_{2}+i y\right)$ with $y=0$ corresponds to the line (the ray) in $\mathbb{R}^{3}$ passing through the origin and having the direction vector $\left\langle\mu_{2}, 1, \mu_{1}\right\rangle$. Now, a group element $g=\left(\begin{array}{ll}a & b \\ c & d\end{array}\right) \in \mathbf{S L}(2, \mathbb{C})$ acting on nonzero vectors $\left(\begin{array}{l}z_{1} \\ z_{2}\end{array}\right) \in \mathbb{C}^{2}$ induces the action on slopes of the lines $z_{2}=\mu z_{1}$ given by $\mu \longmapsto \mu^{\prime}=\frac{d \mu+c}{b \mu+a}$.

Consequently, $\mathbf{S L}(2, \mathbb{C})$ is acting on the extended image plane $\widehat{\mathbb{C}}=\mathbb{C} \cup$ $\{\infty\}$, where the point $\infty$ corresponds to the line $z_{1}=0$, by linear-fractional transformations (this action agrees with the action in (3)) given by

$$
z \longmapsto\left(\begin{array}{ll}
a & b \\
c & d
\end{array}\right) \cdot z=\frac{d z+c}{b z+a}, \quad \infty \longmapsto\left(\begin{array}{ll}
a & b \\
c & d
\end{array}\right) \cdot \infty=\frac{c}{a} .
$$

Therefore, the extended image plane $\widehat{\mathbb{C}}$ can be identified with the complex projective line $P^{1}(\mathbb{C})=\left\{\right.$ lines in $\mathbb{C}^{2}$ passing through the origin $\}$ where the group of projective transformations (or, homographs of the projective line) is $\mathbf{P S L}(2, \mathbb{C})=\mathbf{S L}(2, \mathbb{C}) /\{ \pm \mathbf{I}\}$. We note that this group is the same as the projective group for patterns derived in the conformal camera model. Further, the stereographic projection $\left.j\right|_{\mathbf{S}_{(0,1,0)}^{2}}$ (with $j$ given in (1)) from the unit sphere centered at $(0,1,0)^{t}$, with the west pole $(0,0,0)^{t}$ projected into $\infty$, shows that $\widehat{\mathbb{C}} \cong \mathbf{S}_{(0,1,0)}^{2}$.

The projective (conformal) camera model and the framework of Fourier analysis on groups imply that the projectively covariant harmonic analysis should be developed upon the irreducible unitary representations of the group $\mathbf{S L}(2, \mathbb{C})$ as the basic building blocks of this analysis.

We now follow [14] to give a nontechnical explanation of this point. Informally, an irreducible representation of a (topological) group $\mathbf{G}$ is a group homomorphism $\mathcal{T}$ (with some continuity requirements) between $\mathbf{G}$ and the group of bounded linear operators on a complex Hilbert space $V$, such that the mapping $\mathcal{T}$ cannot be "broken up" into a direct sum of "smaller" homomorphisms. Intuitively, we can say that the composition of those bounded linear operators mirrors the group operation in G. In fact, all such inequivalent representations (of $\mathbf{G}$ in terms of those linear operators) consist of the 
simplest possible and complete set of building blocks of harmonic analysis on $\mathbf{G}$.

Thus, this framework provides desired projectively covariant representations of images if $\mathbf{G}=\mathbf{S L}(2, \mathbb{C})$ - the projective group for patterns.

We note that representations are unitary if the operators are unitary. It is well-known that if $\mathbf{G}$ is abelian then the operators are one-dimensional (i.e., they are the characters of $\mathbf{G}$ ), and if it is a compact group then the operators are finite-dimensional (i.e., matrices). If the Hilbert space $V$ is infinite-dimensional, the representation is infinite-dimensional.

All the unitary representations of the semisimple Lie group $\mathbf{S L}(2, \mathbb{C})$ are infinite-dimensional [17] and they are constructed in different realizations on the spaces of homogeneous functions on $\mathbb{C}^{2}$, giving different pictures of the projective harmonic analysis. Two such realizations are fundamental in our work. The first one, referred to as the "noncompact picture", is the restriction of the analysis to the affine patch $\mathbb{C}$ of $\widehat{\mathbb{C}}$. The second realization, referred to as the "compact picture", is obtained by working on the unit sphere $\mathbf{S}^{2}$ (which is isomorphic to $\widehat{\mathbb{C}}$ ). The homogeneity of the functions on $\mathbb{C}^{2}$ implies that both restrictions are one-to-one. The reference [17] contains a discussion of these realizations. In the case of Euclidean group, the corresponding restrictions to the noncompact and compact pictures result in the classical and spherical Fourier analyses, respectively, both making up Euclidean Fourier analysis, see $[18]$.

In a more recent approach (which extends to other semisimple Lie groups) the irreducible unitary representations of $\mathbf{S L}(2, \mathbb{C})$ are induced in a one-toone way from the Borel subgroup $\mathbf{B}=$ MAN where $\mathbf{N}=\overline{\mathbf{N}}^{t}$ (the set of all transpose matrices of $\overline{\mathbf{N}}$ where $\overline{\mathbf{N}}$ is given in Section 2.1). This, the so-called, induced picture is based on the two decompositions of $\mathbf{S L}(2, \mathbb{C})$. The first one that is given by

$$
\mathbf{S L}(2, \mathbb{C})=\overline{\mathbf{N B}} \cup p \mathbf{B}, \quad p=\left(\begin{array}{ll}
0 & 1 \\
-1 & 0
\end{array}\right)
$$

implies Gauss decomposition

$$
\mathrm{SL}(2, \mathbb{C}) \doteq \overline{\mathrm{NB}}
$$

where "" means that the equality holds up to a set of measure zero. The 
second one is Iwasawa decomposition

$$
\mathbf{S L}(2, \mathbb{C})=\mathbf{S U}(2) \mathbf{A N}
$$

The one-to-one restrictions of the unitarily induced representations to the subgroups $\overline{\mathbf{N}}$ and $\mathbf{S U}(2)$ result in the noncompact and compact pictures, respectively. A well-motivated survey of this approach can be found in [16].

\section{Projective harmonic analysis in noncom- pact picture}

\subsection{Noncompact picture}

Recall that the noncompact picture of projective Fourier analysis can be constructed by restricting to $\overline{\mathbf{N}} \cong \mathbb{C}$ the unitary representations of $\mathbf{S L}(2, \mathbb{C})$ that are unitarily induced from the finite-dimensional irreducible representations of the Borel subgroup $\mathbf{B}=\mathbf{M A N}$. It follows from Gauss decomposition (9) that this Borel group $\mathbf{B}$ exhausts the projective part of $\mathbf{S L}(2, \mathbb{C})$ since $\overline{\mathbf{N}}$ describes translations in the image plane (which gives the isomorphism $\overline{\mathbf{N}} \cong \mathbb{C}$ mentioned before at the beginning on this section).

It is well-known that the finite-dimensional irreducible unitary representations of the Borel subgroup B are one-dimensional. Indeed, they all are given by the irreducible unitary representation $T^{k, i s} ; k \in \mathbb{Z}, s \in \mathbb{R}$, acting on the Hilbert space $\mathbb{C}$ by $T^{k, i s}(b) \xi=\pi_{k, s}(b) \xi$ where

$$
\pi_{k, s}(b)=\left(\frac{z}{|z|}\right)^{k}|z|^{i s} \quad \text { for } \quad b=\left(\begin{array}{cc}
z & \beta \\
0 & z^{-1}
\end{array}\right)
$$

are called the Borel characters. These representations can be obtained by

extending to $\mathbf{B}$ the characters $\pi_{k, s}: \mathbf{M A} \rightarrow \mathbb{C}, \pi_{k, s}\left(\left(\begin{array}{cc}z & 0 \\ 0 & z^{-1}\end{array}\right)\right)=\left(\frac{z}{|z|}\right)^{k}|z|^{i s}$. Also, because $\mathbf{M A} \subset \mathbf{S L}(2, \mathbb{C})$ is topologically isomorphic with the multiplicative group of nonzero complex numbers $\mathbb{C}_{*}, \pi_{k, s}(z)=\left(\frac{z}{|z|}\right)^{k}|z|^{i s}$ are the characters of the group $\mathbb{C}_{*}$. 


\section{$3.2 \quad$ Projective Fourier analysis on $L^{2}(\mathbb{C})$}

The classical (Euclidean) Fourier transform on $L^{2}\left(\mathbb{R}^{2}\right)$ is defined in terms of the characters $\gamma_{k}: \mathbb{R}^{2} \rightarrow \mathbb{C}, \gamma_{k}(x)=e^{i k \cdot x} ; k \in \mathbb{R}^{2}$, of the translation (abelian) subgroup $\mathbb{R}^{2}$ of Euclidean (semidirect product) group $\mathbf{S O}(2) \overline{\times} \mathbb{R}^{2}$. Similarly, the projective Fourier transform in noncompact picture is defined in terms of the characters of the abelian subgroup $\mathbf{M A} \cong \mathbb{C}_{*}$, and therefore, in terms of the finite-dimensional irreducible unitary representations of the subgroup $\mathbf{B}$ of $\mathbf{S L}(2, \mathbb{C})$ (recall Section 3.1). For a given intensity function $f(z)$ in (5), its projective Fourier transform is defined as follows

$$
\widehat{f}(k, s)=\frac{i}{2} \int f(z)\left(\frac{z}{|z|}\right)^{-k}|z|^{-i s-1} d z d \bar{z}
$$

where the extra factor $|z|^{-1}$ unitarizes the transformation. We note that if $z=x+i y$, then $(i / 2) d z d \bar{z}=d x d y$.

We have obtained in [11] the inverse projective Fourier transform in this picture,

$$
f(z)=(2 \pi)^{-2} \sum_{k=-\infty}^{\infty} \int_{-\infty}^{\infty}|z|^{i s-1}\left(\frac{z}{|z|}\right)^{k} \widehat{f}(k, s) d s,
$$

and the corresponding Plancherel theorem which says that for $f \in L^{2}(\mathbb{C})$ we have $\|f\|_{2}=|\| \widehat{f}|||$ where $\|f\|_{2}^{2}=\frac{i}{2} \int_{\mathbb{C}}|f(x)|^{2} d z d \bar{z}$ and $\left.|\| \widehat{f}|\right|^{2}=\int|\widehat{f}(k, s)|^{2} d \omega$ with the measure $d \omega$ given by

$$
\int F(k, s) d \omega=(2 \pi)^{-2} \sum_{k=-\infty}^{\infty} \int_{-\infty}^{\infty} F(k, s) d s .
$$

We mention that by Plancherel's theorem both the transform and its inverse extend to the space of square-integrable functions and therefore the statements such as "the equality holds up to a set of measure zero" made for (9) are acceptable.

The convolution in noncompact picture is defined on the subgroup MA by

$$
f_{1} * f_{2}(z)=\frac{i}{2} \int f_{1}\left(g^{-1} \cdot z\right) f_{2}(\xi) \frac{d \xi d \bar{\xi}}{|\xi|^{2}}
$$


where

$$
g=\left(\begin{array}{cc}
\delta^{-1 / 2} e^{-i \varphi / 2} & 0 \\
0 & \delta^{1 / 2} e^{i \varphi / 2}
\end{array}\right) \quad \text { and } \quad \xi=\delta e^{i \varphi} .
$$

Taking the projective Fourier transform of the convolution (14) and changing the variable by $\eta=\xi^{-1} z$, we easily obtain the convolution property:

$$
\widehat{f_{1} * f_{2}}(k, s)=\widehat{f_{1}}(k, s) \widehat{f_{2}}(k, s) .
$$

\section{Discrete Projective Fourier Transform in Noncompact Picture}

On introducing $\xi=e^{u+i \theta}$, where $r=e^{u}$ and $\theta$ are polar coordinates, into (12), we express this integral as the standard Fourier integral

$$
\widehat{f}(k, s)=\int_{-\infty}^{\infty} \int_{0}^{2 \pi} e^{u} f\left(e^{u+i \theta}\right) e^{-i(u s+\theta k)} d \theta d u .
$$

Then, we take the set $D_{s}=[0, T] \times[0,2 \pi / L]$ as the domain of the function $g(u, \theta)=f\left(e^{u+i \theta}\right) e^{u}$ and extend periodically the function in the $u$-coordinate by $g(u+m T, \theta)=g(u, \theta)$ where $m \in \mathbb{Z}$. This implies the following condition on the extension;

$$
f\left(e^{u+m T+i k \theta}\right)=f\left(e^{u+i k \theta}\right) e^{-m T} .
$$

Next, we define the function $h$ by $h(\vartheta, \gamma)=g(u, \theta)=g\left(\frac{\vartheta T}{2 \pi}, \frac{\gamma}{L}\right)$ which is $2 \pi$-periodic with respect to both variables $\vartheta$ and $\gamma$, and therefore, it can be expanded in a double Fourier series. Expressing this series in terms of $g(u, \theta)$ rather than $h(\vartheta, \gamma)$, we obtain (see [12]),

$$
\widehat{f}(2 \pi m / T, n L)=\int_{0}^{T} \int_{0}^{2 \pi / L} g(u, \theta) e^{-i(2 \pi m u / T+n \theta L)} d \theta d u
$$

and

$$
g(u, \theta)=\frac{L}{2 \pi T} \sum_{m=-\infty}^{\infty} \sum_{n=-\infty}^{\infty} \widehat{f}(2 \pi m / T, n L) e^{i(2 \pi m u / T+n \theta L)}
$$


Further, we approximate the integral in (16) by a double Riemann sum,

$$
\sum_{k=0}^{M-1} \sum_{l=0}^{N-1} \frac{2 \pi T}{L N M} g(k T / M, l 2 \pi / L N) e^{-2 \pi i(m k / M+n l / N)},
$$

where the interval $[0, T]$ is divided into $M$ subintervals by points $u_{k}=k T / M$ $(k=0, \ldots, M-1)$ and the interval $[0,2 \pi / L]$ into $N$ subintervals by points $\theta_{l}=l 2 \pi / L N(l=0, \ldots, N-1)$.

Finally, defining $f_{k, l}$ by

$$
f_{k, l} e^{k T / M}=\frac{2 \pi T}{L N M} g(k T / M, l 2 \pi / L N)
$$

and

$$
\widehat{f}_{m, n}=\sum_{k=0}^{M-1} \sum_{l=0}^{N-1} f_{k, l} e^{k T / M} e^{-2 \pi i(k m / M+l n / N)}
$$

(which is doubly periodic with period $\left.(M, N): \widehat{f}_{m+M, n+N}=\widehat{f}_{m, n}\right)$ and introducing the relation $z_{m, n}=e^{m T / M} e^{i \frac{2 \pi n}{L N}}=r_{m} e^{i \theta n}$, we arrive at

$$
\widehat{f}_{m, n}=\sum_{k=0}^{M-1 N-1} \sum_{l=0} f_{k, l}\left(\frac{z_{k, l}}{\left|z_{k, l}\right|}\right)^{-n L}\left|z_{k, l}\right|^{-i 2 \pi m / T+1}
$$

and

$$
f_{k, l}=\frac{1}{M N} \sum_{m=0}^{M-1 N-1} \sum_{n=0} \widehat{f}_{m, n}\left(\frac{z_{k, l}}{\left|z_{k, l}\right|}\right)^{n L}\left|z_{k, l}\right|^{i 2 \pi m / T-1}
$$

for functions $f_{k, l}$ satisfying $f_{k+m M, l+n L}=f_{k, l} e^{-m T}$ where $m, n \in \mathbb{Z}$.

The expressions in (18) and (19) are called the $(M, N)$-point discrete projective Fourier transform and its inverse, respectively.

\section{Projective harmonic analysis in compact picture}

\subsection{Compact picture}

Iwasawa decomposition (10) implies that

$$
\mathbf{S L}(2, \mathbb{C}) / \mathbf{B} \cong \mathbf{S U}(2) / \mathbf{M} \text {. }
$$


where $\mathbf{B}=\mathbf{M A N}$ is the Borel subgroup. The compact picture is referred to harmonic analysis of patterns restricted to the subgroup $\mathbf{S U}(2)$ which is acting on its homogeneous space $\mathbf{S U}(2) / \mathbf{M}$. By (8), $\mathbf{S U}(2) / \mathbf{M}$ is isomorphic to $P^{1}(\mathbb{C})$, and hence also to $\mathbf{S}_{(0,1,0)}^{2}$.

Further, recalling that $\mathbf{S U}(2)$ is the universal double cover of $\mathbf{S O}(3)$ (cf., [11]) and using Euler's angle parametrization of $\mathbf{S U}(2)$, we have the diagram

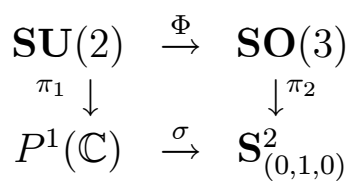

where $\pi_{1}$ is the projection onto the quotient space $\mathbf{S U}(2) / \mathbf{M} \cong P^{1}(\mathbb{C}), \pi_{2}$ is the projection onto $\mathbf{S O}(3) / \mathbf{S O}(2) \cong \mathbf{S}_{(0,1,0)}^{2}$ and $\Phi\left(k\left(\psi, \phi, \psi^{\prime}\right)\right)=R\left(\psi, \phi, \psi^{\prime}\right)$ with $k\left(\psi, \phi, \psi^{\prime}\right)$ given in (2). Also, the mapping $\sigma=\left(\left.j\right|_{\mathbf{S}_{(0,1,0)}^{2}}\right)^{-1}$ is the inverse of the stereographic projection of the unit sphere $\mathbf{S}_{(0,1,0)}^{2}$ onto the plane $x_{2}=1$, given explicitly by

$$
\sigma(z)=\left(\frac{2 \operatorname{Im} z}{|z|^{2}+1}, \frac{2}{|z|^{2}+1}, \frac{2 \operatorname{Re} z}{|z|^{2}+1}\right)^{t}
$$

where $\operatorname{Im} z$ and $\operatorname{Re} z$ stand for the imaginary and real parts of $z$, respectively.

Now, one can verify, see [13], that the homomorphisms $\Phi$ and $\sigma$ in (21) satisfy $\Phi(k) \circ \sigma=\sigma \circ k$ with both sides acting on $P^{1}(\mathbb{C})$. This is used in the next section to formulate projective Fourier analysis in the compact picture by taking the pull back of spherical Fourier analysis by $(\Phi, \sigma)$ in the diagram (21).

\subsection{Projective Fourier analysis on $L^{2}\left(\mathbb{C}, \frac{2 i}{\left(1+|z|^{2}\right)^{2}} d z d \bar{z}\right)$}

From the homomorphism $\Phi$ in (21) and spherical harmonic analysis (for example, see [19]), it follows that the irreducible unitary representations of the group $\mathbf{S U}(2)$ on $L^{2}(\mathbf{S U}(2) / \mathbf{M})$ are $\mathcal{R}^{(l)}=\mathcal{T}^{(l)} \circ \Phi ; l \in \mathbb{N}$, where $\mathcal{T}^{(l)}$ is the irreducible unitary representation of $\mathbf{S O}(3)$ of dimension $2 l+1$. (It is well-known that the representation $\mathcal{R}^{(l)}$ of $\mathbf{S U}(2)$ defines the representation $\mathcal{T}^{(l)}$ of $\mathbf{S O}(3)$ by means of formula $\mathcal{T}^{(l)} \circ \Phi=\mathcal{R}^{(l)}$ if and only if $l$ is a natural 
number.) We recall from spherical harmonic analysis that in the orthonormal basis $\left\{Y_{l}^{m} ; l \in \mathbb{N},-l \leq m \leq l\right\}$ of spherical harmonics,

$$
Y_{l}^{m}(\psi, \phi)=(-1)^{m} \sqrt{\frac{(2 l+1)(l-m) !}{4 \pi(l+m) !}} P_{l}^{m}(\cos \phi) e^{i m \psi},
$$

where $P_{l}^{m}$ are the associated Legandre polynomials [19], the sum on the right hand side in

$$
\mathcal{T}(R) Y_{l}^{m}=\sum_{|k| \leq l} Y_{l}^{k} T_{k, m}^{(l)}(R)
$$

provides the decomposition of the regular representation $\mathcal{T}(R) f(\omega)=f\left(R^{-1} \omega\right)$ into irreducible unitary representations $\mathcal{T}^{(l)}(R)=\left(T_{m, n}^{(l)}\right)(R)$. Here, for $R$ $=R\left(\psi, \phi, \psi^{\prime}\right) \in \mathbf{S O}(3), T_{m, n}^{(l)}=e^{-i m \psi} t_{m, n}^{(l)}(\cos \phi) e^{-i n \psi^{\prime}}$ with $t_{m, n}^{(l)}$ related to Jacobi polynomials [19]. In particular,

$$
T_{m, 0}^{(l)}=\sqrt{\frac{4 \pi}{2 l+1}} \bar{Y}_{l}^{m}
$$

are the spherical functions.

Thus, the harmonic decomposition of functions on $P^{1}(\mathbb{C}) \cong \mathbf{S U}(2) / \mathbf{M}$ will be given with respect to, what we call, the projective harmonics $Z_{l}^{m}=$ $Y_{l}^{m} \circ \sigma$, explicitly given by

$$
Z_{l}^{m}(z)=(-1)^{m} \sqrt{\frac{(2 l+1)(l-m) !}{4 \pi(l+m) !}} P_{l}^{m}\left(\frac{1-|z|^{2}}{1+|z|^{2}}\right) e^{i m \arctan \left(\frac{\operatorname{Im} z}{\operatorname{Re} z}\right)} .
$$

To this end, we first note that from (2) we have the factorization $k=$ $m(\psi / 2) t(\phi / 2) m\left(\psi^{\prime} / 2\right)$ where

$$
m(\psi / 2)=\left(\begin{array}{cc}
e^{i \phi / 2} & 0 \\
0 & e^{-i \phi / 2}
\end{array}\right)
$$

and

$$
t(\phi / 2)=\left(\begin{array}{cc}
\cos \phi / 2 & i \sin \phi / 2 \\
i \sin \phi / 2 & \cos \phi / 2
\end{array}\right)
$$


Then, one can easily check that the subgroup $\mathbf{M}$ of elements $m(\psi / 2)$ fixes the point 0 and its action on the image plane $\mathbb{C}$ consists in rotations of angle $\psi$ about the origin, and the subgroup $\mathbf{T}$ of elements $t(\phi / 2)$ moves the point 0 of $\mathbb{C}$ along the positive imaginary axis for $0 \leq \phi<\pi$. It provides the parametrization $z=\tan \frac{\phi}{2} e^{i \psi}$ of the image plane by polar coordinates expressed in terms of the Euler angles. Now, the pullback by the mapping $\sigma$ : $\mathbb{C} \rightarrow \mathbf{S}_{(0,1,0)}^{2}$ of the rotational-invariant measure $d \omega=\sin \phi d \phi d \psi$ on the unit sphere is the $\mathbf{S U}(2)$-invariant measure $d p:=\sigma^{*}(d \omega)=2 i\left(1+|z|^{2}\right)^{-2} d z d \bar{z}$ on $\mathbb{C}$. To this end, we observe that if $z^{\prime}=g^{-1} \cdot z$ where $g=\left(\begin{array}{cc}\bar{a} & -\bar{b} \\ c & d\end{array}\right) \in \mathbf{S U}(2)$ then, $d z^{\prime} d \bar{z}^{\prime}=|\bar{b} z+a|^{-4} d z d \bar{z}$. Next, we verify $1+\left|z^{\prime}\right|^{2}=|\bar{b} z+a|^{-2}\left(1+|z|^{2}\right)$. It implies $\left(1+\left|z^{\prime}\right|^{2}\right)^{-2} d z^{\prime} d \bar{z}^{\prime}=\left(1+|z|^{2}\right)^{-2} d z d \bar{z}$, which is the desired invariance property.

Choosing the basis of $2 l+1$ spherical harmonics $Y_{l}^{m} ;-l \leq m \leq l$, for each $l \geq 0$ gives an orthonormal basis $Z_{l}^{m}(z) ;-l \leq m \leq l$, for all of $L^{2}(\mathbb{C}, d p)$ and in analogy with the harmonic analysis on $L^{2}(\mathbf{S O}(3) / \mathbf{S O}(2))$, we obtain the projective harmonic decomposition of $f \in L^{2}(\mathbb{C}, d p)$ in the form

$$
f(z)=\sum_{l \in \mathbb{N}} \sum_{|m| \leq l} \widehat{f}(l, m) Z_{l}^{m}(z)
$$

where the coefficients $\widehat{f}(l, m)$ of the decomposition are given by the Fourier transform

$$
\widehat{f}(l, m)=\int f(z) \bar{Z}_{l}^{m}(z) 2 i\left(1+|z|^{2}\right)^{-2} d z d \bar{z}
$$

We call (28) the projective Fourier transform in compact picture. Its inverse transform is given in (27). The next theorem [13] summarizes the projectively covariant characteristics of the harmonic decomposition in the compact picture.

Theorem 1 The decomposition

$$
f(z)=\sum_{l \in \mathbb{N}} \sum_{|m| \leq l} \widehat{f}(l, m) Z_{l}^{m}(z)
$$

under the projective transformation $\mathcal{R}(g) f(z)=f\left(g^{-1} \cdot z\right), g \in \mathbf{S U}(2)$ trans- 
forms as follows

$$
\mathcal{R}(g) f(z)=\sum_{l \in \mathbb{N}} \sum_{|k| \leq l}\left(\sum_{|m| \leq l} \widehat{f}(l, m) \bar{R}_{m, k}^{(l)}\left(g^{-1}\right)\right) Z_{l}^{k}(z)
$$

where for each $l \in \mathbb{N}$

$$
\begin{aligned}
\mathcal{R}^{(l)}(g) & =\left(R_{m, k}^{(l)}(g)\right) \\
& =\left(T_{m, k}^{(l)} \circ \Phi(g)\right)
\end{aligned}
$$

is the irreducible unitary representation of $\mathbf{S U}(2)$ on the Hilbert space $W^{l}=$ $\left\{Z_{l}^{m}(z): l \in \mathbb{N},-l \leq m \leq l\right\}$ with the inner product

$$
\langle f, h\rangle=\int f(z) \bar{h}(z) 2 i\left(1+|z|^{2}\right)^{-2} d z d \bar{z} .
$$

The Plancherel theorem takes on here the following form,

$$
\|\left. f\right|_{L^{2}(\mathbb{C}, d p)} ^{2}=\sum_{l=0}^{\infty} \sum_{k=-l}^{l}|\widehat{f}(l, k)|^{2},
$$

which says that the mapping $f(z) \longmapsto \widehat{f}(l, k)$ is isometric with respect to the corresponding norms. It follows directly from the definition of projective harmonics and the Plancherel theorem in spherical harmonic analysis.

For two functions $f_{1}$ and $f_{2}$ on $\mathbb{C}$ the operator of left convolution by $f_{1}$ is defined by

$$
\begin{aligned}
\mathfrak{C}_{f_{1}} f_{2}(z) & =\int_{\mathbf{S U}(2)} f_{1}(g \cdot 0) \mathcal{T}(g) f_{2}(z) d g \\
& =\int_{\mathbf{S U}(2)} f_{1}(g \cdot 0) f_{2}\left(g^{-1} \cdot z\right) d g \\
& =f_{1} * f_{2}(z) .
\end{aligned}
$$

Since the operators $\mathcal{R}(g)$ are simultaneously block diagonalized for all $g \in$ SU(2) in the projective harmonic basis $Z_{l}^{m}$ (because $\mathcal{T}^{(l)}$ are simultaneously block diagonalized in the basis $Y_{l}^{m}$ ), the convolution operator $\mathfrak{C}_{f_{1}}$ obtained as their linear combination must be block diagonalized as well. More explicitly, we have the following result [13] (which can also be proved along the same lines for right convolution). 
Theorem 2 The convolution property

$$
\widehat{f_{1} * f_{2}}(l, m)=2 \pi\left(\frac{4 \pi}{2 l+1}\right)^{1 / 2} \widehat{f}_{1}(l, m) \widehat{f}_{2}(l, m) .
$$

\section{$6 \quad$ Numerical test and conclusions}

There are two distinct approaches for representing images in machine vision; the geometric way and the imaging way. The former uses geometrical primitives such as lines, polygons or splines to represents shapes and extract invariants. The latter, on the other hand, deals with arrays of numbers - discrete samples of pixels - that are coming often from non-geometrical sources such as digitized satellite photographs or X-radiographs. Of course, both approaches are interrelated since one can extract geometrical data from sampled data. However, it introduces thresholding artifacts that are highly undesirable in many situations such as medical diagnostic imaging, for example.

The cameras used in computational vision belong to the geometric approach. On the other hand, the $\operatorname{PSL}(2, \mathbb{C})$-camera model constructed in this work, which justifies the word "projective" in the case of planar objects (i.e., patterns), belongs to the imaging approach.

Nevertheless, this camera introduces conformal distortions of image perspective transformations. To see this, we recall from Section 2.1 that $j \circ$ $R \circ\left(\left.j\right|_{\mathbf{S}_{(0,1,0)}^{2}}\right)^{-1}=k \in \mathbf{S U}(2)$ implies that a pattern on the image plane $x_{2}=1$ is first projected by $\left(\left.j\right|_{\mathbf{S}_{(0,1,0)}^{2}}\right)^{-1}$ (the inverse of stereographic projection with $j$ given in (1)) into the sphere $\mathbf{S}_{(0,1,0)}^{2}$ which then is rotated by $R(\psi) R(\phi) R\left(\psi^{\prime}\right) \in \mathbf{S O}(3)$ and finally projected back into the image plane, recall Figure 1. For example, taking the line segment in the image plane along the $x_{3}$-axis from $(0,1,0)$ to $(0,1,1)$ and applying $j \circ R \circ\left(\left.j\right|_{\mathbf{S}_{(0,1,0)}^{2}}\right)^{-1}$ to it, where $R=R(\psi) R(\phi) R\left(\psi^{\prime}\right)$ with $\psi=\psi^{\prime}=0$ and $\phi=\frac{\pi}{2}$, the image of this line segment produced this way is the unit circle circumference segment. The reader should consult Figure 1 to see that it is the case. In order to render image perspective transformations of a pattern as produced by this camera, one must remove those distortions. These are in fact conformal distortions, i.e., distortions preserving angles, since stereographic projections and linear fractional transformations are conformal mappings [20]. 
This "conformal lens" problem of the $\mathbf{P S L}(2, \mathbb{C})$-camera is somewhat similar to the problem of the absence of optical lenses in a pinhole camera. This camera model, while being a useful starting point in computational vision, needs ex posto facto calibration to account for effects of optical lenses of imaging systems, see [21]. In fact, a real lens of optical system does not produce a uniform mapping across the field of view. There are radial distortions which become more severe with radial distance from the principal point, the point where the optical axis intersects the image plane.

Moreover, the PSL $(2, \mathbb{C})$-camera model can develop also some artificial global (topological) effects as far as image perspective transformations of planar objects are considered. One can easily get the idea of such problems by taking a thin ring pattern of inner radius equal to, say, 1 and its center at the origin and applied the inversion transformation $z \longmapsto \frac{1}{z}$ to it $(\phi=\pi$ and $\psi=\psi^{\prime}=0$ in (3) ), the transformation that is gluing to copies of $\mathbb{C}$ to produce $P^{1}(\mathbb{C})$, see [11]. This image transformation interchanges the outer and inner boundaries of the ring pattern. Of course, the image plane $\mathbb{C}$ and the Riemann sphere $\widehat{\mathbb{C}}$ have different topological characteristics and such effects should not be totally unexpected.

\subsection{Rendering image perspective transformations}

The image projective transformations (6) can be rendered by calculating only the projective Fourier transform of the intensity function $f$ of the original pattern (5) as follows. If $z^{\prime}=g^{-1} \cdot z, g \in \mathbf{S L}(2, \mathbb{C})$, then by (13) we have

$$
\begin{aligned}
f\left(g^{-1} \cdot z\right) & =f\left(z^{\prime}\right) \\
& =(2 \pi)^{-2} \sum_{k=-\infty}^{\infty} \int_{-\infty}^{\infty} \widehat{f}(k, s)\left(\frac{z^{\prime}}{\left|z^{\prime}\right|}\right)^{k}\left|z^{\prime}\right|^{i s-1} d s .
\end{aligned}
$$

where $\widehat{f}(k, s)$ is the projective Fourier transform of $f(z)$ and is given in (12).

In a numerical test (see $[11,13,15]$ for more numerical tests), following the method described in [11], we simulate projective distortions of a ring pattern shown in Figure 2 (a). Image projective transformations of the pattern are obtained by rendering (32) with

$$
g=\left(\begin{array}{cc}
e^{-i \psi / 2} \cos \frac{\phi}{2} & i e^{-i \psi / 2} \sin \frac{\phi}{2} \\
i e^{i \psi / 2} \sin \frac{\phi}{2} & e^{i \psi / 2} \cos \frac{\phi}{2}
\end{array}\right) \in \mathbf{S U}(2),
$$


by running Maple's procedure "densityplot" for the approximation

$$
\sum_{k=-N}^{N} \int_{-S}^{S}\left|z^{\prime}\right|^{i s-1}\left(\frac{z^{\prime}}{\left|z^{\prime}\right|}\right)^{k} \widehat{f}(k, s) d s \approx \sum_{k=-\infty}^{\infty} \int_{-\infty}^{\infty}\left|z^{\prime}\right|^{i s-1}\left(\frac{z^{\prime}}{\left|z^{\prime}\right|}\right)^{k} \widehat{f}(k, s) d s
$$

where

$$
z^{\prime}=g^{-1} \cdot z=\frac{z e^{-i \psi / 2} \cos \frac{\phi}{2}-i e^{-i \psi / 2} \sin \frac{\phi}{2}}{-i z e^{i \psi / 2} \sin \frac{\phi}{2}+e^{i \psi / 2} \cos \frac{\phi}{2}}
$$

and $N=120$ and $S=220$. This densityplot simulates the image "intensity" function of the approximation which is treated as a function of the image points $z$.

In Figure 2 (a) both $\phi$ and $\psi$ in (33) are zero, which means that the ring pattern shown there is rendered by using the inverse projective Fourier transform (that is, it is a reconstruction problem). In (b) we render the image projective transformation of the ring pattern by applying (33) in (34) with $\phi=45^{\circ}$ and $\psi=60^{\circ}$. Recalling the $\operatorname{PSL}(2, \mathbb{C})$-camera model in Figure 1 , one sees that this projective deformation is produced by rotating the pattern's projection on the sphere $\mathbf{S}_{(0,1,0)}^{2}$ by $45^{\circ}$ about the axis parallel to the $x_{3}$-axis and passing through $(0,1,0)$, followed by the rotation about the $x_{2}$-axis by $60^{\circ}$ (the latter is just the rotation in the image plane). The following image projective transformations in (c) and (d) are produced in the same way by the indicated Euler's angles. The deformation in (e) is rendered with the value of $\psi$ as for the deformation in (d) in order to focus on the effect of the deformation sequence passing through the point at infinity. The last simulation in $(\mathrm{h})$ shows the pattern obtained from the pattern in (a) by applying the corresponding transformation $z \longmapsto \frac{1}{z}$ (recall the discussion in the previous subsection). Thus, we have rendered the pattern's image perspective transformations using only one projective Fourier transform of the original pattern. 


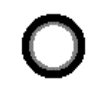

(a) $\phi=0, \psi=0$

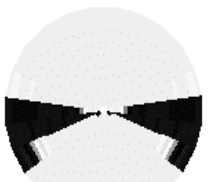

(d) $\phi=94.7^{\circ}, \psi=180^{\circ}$

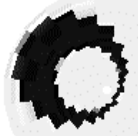

(b) $\phi=45^{\circ}, \psi=60^{\circ}$

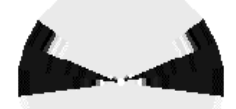

(e) $\phi=100^{\circ}, \psi=180^{\circ}$

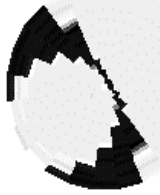

(c) $\phi=66.7^{\circ}, \psi=120^{\circ}$

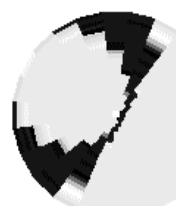

(f) $\phi=112.5^{\circ}, \psi=240^{\circ}$

O

(g) $\phi=138.5^{\circ}, \psi=300^{\circ}$

(h) $\phi=180^{\circ}, \psi=360^{\circ}$

Fig. 2. Computer simulations of the image projective transformations of the ring pattern reconstructed in (a). The discussion is given in the text.

\subsection{On fast algorithms}

The covariant characteristics are preserved by the discrete inverse transform (19), as for any $g \in \mathbf{S L}(2, \mathbb{C})$

$$
f_{m, n}^{\prime}=\frac{1}{M N} \sum_{k=0}^{M-1 N-1} \sum_{l=0}^{N} \widehat{f}_{k, l}\left(\frac{g^{-1} \cdot z_{m, n}}{\left|g^{-1} \cdot z_{m, n}\right|}\right)^{n L}\left|g^{-1} \cdot z_{m, n}\right|^{2 \pi k m / T-1},
$$


where $f_{m, n}^{\prime}=\frac{2 \pi T}{L M N} f\left(g^{-1} \cdot z_{m, n}\right)$ is the corresponding projective transformation of the sampled values $f_{m, n}$ of the pattern $f\left(z_{m, n}\right)=\frac{L M N}{2 \pi T} f_{m, n}$.

This transformation is expressed in (35) only in terms of the projective discrete Fourier transform $\widehat{f}_{k, l}$ of the original pattern $f_{m, n}$ that is given in $(u, \theta)$-coordinates by

$$
\widehat{f}_{k, l}=\sum_{m=0}^{M-1 N-1} \sum_{n=0}^{N} f_{m, n} e^{u_{m}} e^{-i 2 \pi k u_{m} / T} e^{-i \theta_{n} l L} .
$$

Here, $r_{m}=e^{u_{m}}$ and $\left(u_{m}, \theta_{n}\right)$, where $u_{m}=\frac{m T}{M}$ and $\theta_{n}=\frac{2 \pi n}{L N}$, are equally spaced points in the set $D^{\prime}=[0, T] \times[0,2 \pi / L]$. Thus $\left[\widehat{f}_{k, l}\right]$ can by computed by applying 2-D FFT algorithms, see also [12].

How to compute efficiently the projectively distorted pattern $f_{m, n}^{\prime}$, represented in (35) in terms of $\left[\widehat{f}_{k, l}\right]$ ? To discuss this, we take a sampled point $z_{m, n}=r_{m} e^{i \theta_{n}}$ and recall (see (34)) that its image transformation is given by

$$
z_{m, n}^{\prime}=g^{-1} \cdot z_{m, n}=\frac{z_{m, n} \cos \frac{\phi}{2}-i \sin \frac{\phi}{2}}{-i z_{m, n} \sin \frac{\phi}{2}+\cos \frac{\phi}{2}} .
$$

One can check that under this transformation, the equally spaced points $\left(u_{m}, \theta_{n}\right)$ transform into $\left(u_{m, n}^{\prime}, \theta_{m, n}^{\prime}\right)$ with the coordinates satisfying the equations

$$
e^{2 u_{m, n}^{\prime}}=\frac{e^{u_{m}} \cos ^{2} \frac{\phi}{2}+\sin ^{2} \frac{\phi}{2}-e^{u_{m}} \sin \phi \sin \theta_{n}}{e^{u_{m}} \sin ^{2} \frac{\phi}{2}+\cos ^{2} \frac{\phi}{2}+e^{u_{m}} \sin \phi \sin \theta_{n}}
$$

and

$$
\tan \theta_{m, n}^{\prime}=\frac{1 / 2\left(e^{2 u_{m}}-1\right) \sin \phi+e^{u_{m}} \sin \theta_{n} \cos \phi}{e^{u_{m}} \cos \theta_{n}} .
$$

In terms of $\left(u_{m, n}^{\prime}, \theta_{m, n}^{\prime}\right),(35)$ is now expressed by

$$
f_{m, n}^{\prime}:=\frac{1}{M N} \sum_{k=0}^{M-1} \sum_{l=0}^{N-1} \widehat{f}_{k, l} e^{-u_{m, n}^{\prime}} e^{i 2 \pi u_{m, n}^{\prime} k / T} e^{i \theta_{m, n}^{\prime} l L} .
$$

However, equally spaced property of the sampled points has been destroyed. It seems that the recent advances on irregular sampling theory [22, 23] allow the development of such efficient algorithms. 
In the compact picture, the projective Fourier transforms and convolutions might be efficiently calculated by adapting the fast algorithms developed in [19, 24] for spherical harmonic analysis. However, it might turn out that the fast algorithms could be easier developed directly for projective harmonics.

Then, algorithms for efficient computation of the convolutions in noncompact and compact pictures would follow from the both fast projective Fourier transforms and their inverses. This would be the basis for developing projectively invariant pattern matching algorithms. To this end, we start by recalling the pattern matching problems in Euclidean geometry. Firstly, the two-dimensional convolution on the plane can by efficiently computed by the (standard two-dimensional) FFT. By convolving an image with a pattern, one can locate translated copies of the pattern in the image. Secondly, efficient spherical convolution can be used for pattern matching with directional data. The convolutions in both pictures of the projective Fourier analysis developed in this work involve the corresponding subgroups of the projective group for patterns. In this way they should be useful in located patterns in projectively transformed images, the process done effortlessly by people. It is believed by the author that the efficient algorithms for computing projective convolutions can be used to develop a real time automated system for pattern recognition independent of different perspectives between the planar objects (i.e., patterns) and the imaging system.

\section{Summary}

The inverse projective Fourier transform in noncompact picture (13) provides the decomposition of patterns in terms of the characters of the (noncompact and abelian) group $\mathbb{C}_{*} \cong \mathbf{M A} \subset \mathbf{S L}(2, \mathbb{C})$ (which are also all finitedimensional irreducible unitary representations of $\mathbf{B}=\mathbf{M A N}$, the subgroup which exhausts the projective part of $\mathbf{S L}(2, \mathbb{C})$ ) with the coefficients given by the projective Fourier transform (12). On the other hand, the decomposition in the compact picture (27) is given in terms of projective harmonics (26), with the coefficients given by the corresponding projective Fourier transform (28). Here the projective harmonics are the matrix elements of finite-dimensional unitary representations (expressed in the orthonormal basis providing by these projective harmonics) of the (compact and nonabelian) 
subgroup $\mathbf{S U}(2) \subset \mathbf{S L}(2, \mathbb{C})$, the universal double cover of the rotation group $\mathrm{SO}(3)$.

Because the irreducible representations are the simplest operators that mirrors the projective group operations, both decompositions provide projectively adapted pattern representations, computable by fast algorithms. Moreover, the convolution in the noncompact picture is defined over the subgroup consisting of translations in the optical axis direction $(\mathbf{A})$ and rotations in the image plane of a camera $(\mathbf{M} \subset \mathbf{S U}(2))$, whereas the convolution in the compact picture is defined over the subgroup of all rotations of a camera $(\mathbf{S U}(2))$. Both subgroups together generate the projective group since $\mathbf{S L}(2, \mathbb{C})=\mathbf{S U}(2) \mathbf{A S U}(2)$.

Also, fast algorithms for computing with discrete projective Fourier transforms and for rendering image projective transformations could be developed as demonstrated by the preliminary results. These algorithms can be applied to the efficient computation of convolutions in both pictures. It should be useful in developing perspectively invariant pattern matching, in a similar

way as the convolutions of Euclidean Fourier analysis have been used to locate in images translated and rotated copies of a pattern.

Thus, both pictures of projective Fourier analysis provide the data structure for representing visual inputs, well-suited for developing algorithms for storing and processing visual information, that can be used in automated systems for perspectively independent pattern identification and recognition.

Acknowledgment. This work is supported in part by NSF grant CCR9901957.

\section{References}

[1] N. Blatt and J. Rubinstein, The Canonical Coordinates Method for Pattern Recognition-II. Isomorphism with Affine Transformations, Pattern Recognition 27, 99-107 (1994).

[2] T.M. Cealli and Z-Q. Liu, On the Minimum Number of Templates Required for Shift, Rotation and Size Invariant Pattern Recognition, Pattern Recognition 21, 205-216 (1988). 
[3] M. Ferraro and T.M. Cealli, Relationship Between Integral Transform Invariances and Lie Group Theory, J. Opt, Soc. Amer. A5, 738-742 (1988).

[4] J.-P. Gauthier, G. Bornard and M. Silbermann, Motions and Pattern Analysis: Harmonic Analysis on Groups and Their Homogeneous Spaces, IEEE Trans. Syst. Man Cybernet. 21, 149-172 (1991).

[5] F. Giulianini, M. Ferraro and T.M. Caelli, Transformational Properties of Integral Transforms of Images, J. Opt, Soc. Amer. A9, 494-496 (1992).

[6] F. Ghorbel, A complete Invariant Description for Gray-level Images by the Harmonic Analysis Approach, Pattern Recognition Letters 15, 10431051 (1994).

[7] J. Rubinstein, J. Segman and Y. Zeevi, Recognition of Distorted Patterns by Invariant Kernels, Pattern Recognition 24, 959-967 (1991).

[8] J. Segman, J. Rubinstein and Y.Y. Zeevi, The Canonical Coordinates Method for Pattern Recognition: Theoretical and Computational Considerations, IEEE Trans. Pattern Analysis Mach. Intell. 14, 1171-1183 (1992).

[9] M. Tanaka, On the Representation of the Projected Motion Group in 2+1D, Pattern Recognition Letters 14, 671-678 (1993).

[10] D. Mumford, Mathematical Theories of Shape: Do they model perception? SPIE Vol. 1570 Geometric Methods in Computer Vision, Ed. B.C. Vemuri, 2-10, (1991).

[11] J. Turski, Harmonic Analysis on $\mathbf{S L}(2, \mathbb{C})$ and Projectively Adapted Pattern Representation, J. Fourier Anal. Appl. 4, 67-91 (1998).

[12] J. Turski, Projective Fourier Analysis in Computer Vision: Theory and Computer Simulations, SPIE Vol. 3168 Vision Geometry VI, Eds: R.A. Malter at al., 124-135 (1997).

[13] J. Turski, Projective Fourier Analysis for Computational Vision of Planar Objects, Submitted. 
[14] K.I. Gross, On the Evolution of Noncommutative Harmonic Analysis, Amer. Math. Monthly 85, 525-548 (1977).

[15] J. Turski, Projective Harmonic Analysis and Rendering Perspective Distortions of Patterns, presented at the 4 th International Conference "Curves and Surfaces" held in Saint-Malo, France, July 1-7, 1999.

[16] A.W. Knapp, Representation Theory of Semisimple Groups: An Overview Based on Examples, Princeton Univ. Press, Princeton (1986).

[17] I.M. Gel'fand, M.I. Graev and N.Ja. Vilenkin, Generalized Functions Vol. 5: Integral Geometry and Representations Theory, Academic Press, New York (1966).

[18] E.M. Stein and G. Weiss, Introduction to Fourier Analysis on Euclidean Spaces, Princeton University Press, Princeton (1971).

[19] J.R. Driscoll and D.M. Healy, Computing Fourier Transforms and Convolutions on the 2-Sphere, Advances in Appl. Math. 15, 202-250 (1994).

[20] T. Needham, Visual Complex Analysis, Clarendon Press, Oxford (1997).

[21] J.L. Mundy and A. Zisserman, Appendix - Projective Geometry for Machine Vision, in Geometric Invariance in Computer Vision, Eds: J.L. Mundy and A. Zisserman, 463-519 (1992).

[22] A. Dutt and V. Rokhlin, Fast Fourier Transforms for Nonequispaced Data, II, Appl. Comp. Harmon. Anal. 2, 85-100, (1995).

[23] H.G. Feichtinger, K. Gröchenig and T. Strohmer, Efficient numerical methods in non-uniform sampling theory, Numer. Math. 69, 423-440 (1995).

[24] M.J. Mohlenkamp, A Fast Transform for Spherical Harmonics, J. Fourier Anal. Appl. 5, 159-184 (1999

About the Author - JACEK TURSKI received his Ph.D. from McGill University in 1986 with the dissertation done in both the Departments of Mathematics and Mechanical Engineering. After two years as a 
post-doctoral fellow at the University of Manitoba, he came to the University of Houston as a visiting scholar. Around this time he had developed a research interest in bifurcation theory with symmetries and later started work in the Department of Mathematics on applying this theory to explain pattern formation in superconductors. In 1990 Turski joined the faculty in the Department of Computer and Mathematical Sciences at the University of Houston-Downtown, where now he is holding an associate professor position. For the last four years he has been involved in formulating theory of projective Fourier analysis for patterns. Presently, supported by NSF, he works on developing fast algorithms for computer implementations of this analysis in projectively covariant image processing. 